\title{
Capillary pressure in a porous medium with distinct pore surface and pore volume fractal dimensions
}

\author{
M. R. Deinert \\ Department of Mechanical Engineering, University of Texas at Austin, Austin, Texas 78758, USA \\ A. Dathe \\ Biological and Environmental Engineering, Cornell University, Ithaca, New York 14850, USA \\ J-Y. Parlange \\ Biological and Environmental Engineering, Cornell University, Ithaca, New York 14850, USA \\ K. B. Cady \\ Theoretical and Applied Mechanics, Cornell University, Ithaca, New York 14850, USA
}

(Received 15 December 2007; published 29 February 2008)

\begin{abstract}
The relationship between capillary pressure and saturation in a porous medium often exhibits a power-law dependence. The physical basis for this relation has been substantiated by assuming that capillary pressure is directly related to the pore radius. When the pore space of a medium exhibits fractal structure this approach results in a power-law relation with an exponent of $3-D_{v}$, where $D_{v}$ is the pore volume fractal dimension. However, larger values of the exponent than are realistically allowed by this result have long been known to occur. Using a thermodynamic formulation for equilibrium capillary pressure we show that the standard result is a special case of the more general exponent $\left(3-D_{v}\right) /\left(3-D_{s}\right)$ where $D_{s}$ is the surface fractal dimension of the pores. The analysis reduces to the standard result when $D_{s}=2$, indicating a Euclidean relationship between a pore's surface area and the volume it encloses, and allows for a larger value for the exponent than the standard result when $D_{s}>2$.

DOI: 10.1103/PhysRevE.77.021203

PACS number(s): 65.20.-w, 47.10.-g, 05.20.Jj, 05.45.Df
\end{abstract}

\section{INTRODUCTION}

Accurately predicting capillary pressures in a porous medium is central to understanding the movement and distribution of fluids within such systems. The relationship between these pressures and fluid saturation often follows some form of power law where $\theta / \theta_{s} \simeq\left(P_{s} / P\right)^{\lambda}$ (e.g., [1,2]). Here $\theta$ is the moisture content, $P$ is the capillary pressure, $s$ denotes saturation, and $\lambda$ is an empirically determined exponent. This relation was first established empirically, but subsequent analysis provided it with a physical basis by assuming that both capillary pressure and pore volume are directly proportional to the pore radius. If a system's pore space has fractal structure, this approach then yields a power-law relation between capillary pressure and saturation where the exponent $\lambda$ is equal to $3-D_{v}$, with $D_{v}$ being the pore volume fractal dimension (e.g., [3-8]). However, larger values of the exponent than are realistically allowed by this result have long been known to occur (e.g., [1]).

Recent work on microfluidic cells suggests that it is not so much pore diameter that determines capillary pressure, but fluid interfacial area [9]. Subsequent analysis has shown that the variation in fluid interfacial areas with respect to fluid volume is a significant factor determining capillary pressure under equilibrium conditions [10]. The physical structure of a pore network is particularly relevant in this context because it will directly affect the interfacial areas.

It is well established that the pore space of a wide variety of media exhibits fractal structure (e.g., [11]). It is also known that the surfaces of the grains and particles that com- prise porous systems can themselves be fractal [12,13]. A few studies have recently measured both $D_{v}$ and the surface fractal dimension $D_{s}$ for the same porous medium in an attempt to see if and how they differ. Thin slices from eight different kinds of unconsolidated media, both artificial and natural, were analyzed by Gimenez et al. [14]. Using digital images with a resolution of $60 \mu \mathrm{m}$ they found $D_{v}$ and $D_{s}$ to differ by $0.25-0.56$ with the pore volume fractal dimension exceeding the surface one. Using pulsed gradient NMR, Stallmach et al. [15] found the pore surface and pore volume fractal dimensions of a glacial sand to differ by $0.7 \pm 0.05$. A recent study by Dathe and Thullner [16] analyzed thin slices of two types of undisturbed soil structure using a scanning electron microscope with a resolution of $0.5 \mu \mathrm{m}$ and produced similar results. Other authors have also obtained data showing that distinct fractal dimensions apply to different parts of a porous system (i.e., solid matrix, pore space or grain surface) (e.g., [17-19]).

In the present work we extend our thermodynamic formulation of equilibrium capillary pressure [10] to a system where the pore volume and pore surface fractal dimensions are taken into account. The analysis leads to a power-law relationship between equilibrium capillary pressure and moisture content where the power-law exponent is given by $\left(3-D_{v}\right) /\left(3-D_{s}\right)$. This result allows for the full range of observed exponents and reduces to the standard result when $D_{s}=2$ and gives a larger value for the exponent than the standard result when $D_{s}>2$. The analysis also indicates that a power-law relation between moisture content and capillary pressure will only hold for an equilibrium situation. 


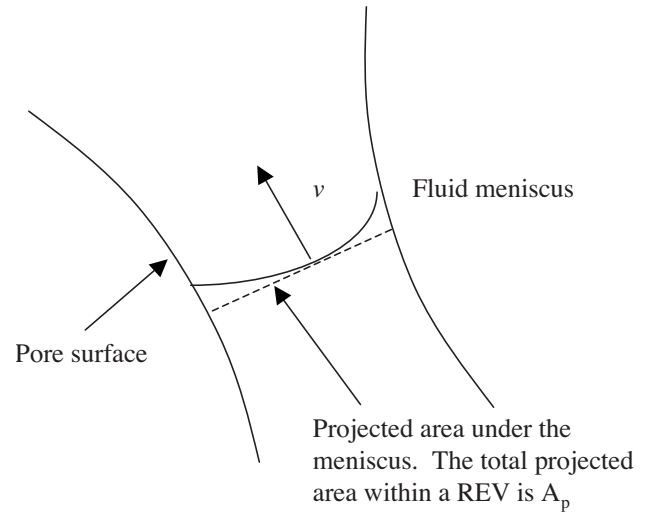

FIG. 1. Fluid meniscus in motion. The last term on the righthand side of Eq. (1) applies to situations where the fluid-solid contact lines with a representative elementary volume (REV) are in motion. Several studies suggest that such movement results in nonequilibrium capillary pressures (e.g., [10,20,21]).

\section{POWER-LAW RELATION BETWEEN CAPILLARY PRESSURE AND SATURATION IN A POROUS MEDIUM}

Using a control mass formulation, the authors previously developed [10] a relation for the internal energy of an element of wetting fluid that enters a region of porous media under pressure $p_{w}$ and distributes itself through a quasistatic process by displacing a nonwetting fluid that is at pressure $p_{n}$ :

$$
U^{\prime}=T S^{\prime}-p_{w} V_{w}^{\prime}-p_{n} V_{n}^{\prime}+\sum \sigma_{w, i} A_{w, i}^{\prime}+\left\langle\alpha \mathbf{F} \cdot \boldsymbol{v} A_{p} v\right\rangle .
$$

Equation (1) adopts the convention that fluid pressure, and related parameters, are quantities averaged over a representative elementary volume (REV) within the soil matrix. Here $U$ is the internal energy, $T$ is the temperature, $S$ is the entropy of the wetting fluid, $p_{w}$ is the pressure in the wetting phase, $p_{n}$ is the pressure in the nonwetting phase, $V_{w}$ is the volume of the wetting fluid, $V_{n}$ is the volume of the nonwetting fluid, and $A_{w, i}$ is the interfacial area of the wetting fluid in contact with the $i$ th nonwetting phase, $\sigma_{w, i}$ is the respective surface tension, and the primes denote the derivative with respect to time. The last term on the right applies to situations where the fluid-solid contact lines with a representative elementary volume (REV) are in motion. Several studies suggest that such movement would result in nonequilibrium capillary pressures (e.g., [10,20,21]). Here $\alpha$ is the total fluid-solid contact line length, $F$ is the force on that line per unit of its length and velocity, $\boldsymbol{v}$ is the velocity of that contact line, $v$ is its magnitude, and $A_{p}$ is the projected interfacial area of the fluid, and the brackets denote an average over a REV, Fig. 1.

Under equilibrium conditions the authors showed [10] that capillary pressure can be written as

$$
P_{c}=-\sum \sigma_{w, i}\left(\partial A_{w, i} / \partial V_{w}\right)_{U, S, T}
$$

At any given saturation in a porous medium under equilibrium conditions, small pores fill first with larger ones doing so when their capillarity can overcome the tension in the fluid surrounding them. A consequence of this in a system with a fractal pore volume distribution is that changes in fluid volume correspond to the filling or emptying of pores at only one scale at a time. In order to evaluate the derivatives in Eq. (1) a relation is therefore needed to give a fluid's interfacial area in terms of its volume within those pores. For a system where the pore surfaces exhibit fractal structure we follow Gimenez et al. [14] and use Mandelbrot's relation between a self similar fractal area and the volume that it encloses ([22], p. 112):

$$
A \propto V^{D_{s} / 3} .
$$

Here $A$ is the surface area of a given pore, $V$ is the volume it encloses, and $2<D_{s}<3$. Equation (3) is the "area-volume" extension of the slit-island relation ([21], p. 112 and [22]) which has previously been shown to hold in a porous medium with fractal pore boundaries [14]. We make the relation in Eq. (3) direct by introducing a constant $c$ such that $A$ $=c V^{D_{s} / 3}$. Assuming that fluid volume is equal to pore volume to a negligible error and that the interfacial areas $A_{w, i}$ follow the proportionality in Eq. (3), $A_{w, i}=f_{w, i} V^{D_{s} / 3}$ where $f_{w, i}$ is a shape factor that remains constant across all scales. Using this relation we can write $\left(\partial A_{w, i} / \partial V_{w}\right)=f_{w, i}\left(D_{s} / 3\right) V^{(1 / 3)\left(D_{s}-3\right)}$, where $V$ is the volume of the largest pore filled. For a situation where a wetting fluid displaces air in a porous medium, Eqs. (1)-(3) can be combined to give

$$
P_{c}=-\left(f_{\text {fluid,air }} \sigma_{\text {fluid,air }}+f_{\text {fluid,solid }} \sigma_{\text {fluid,solid }}\right)\left(D_{s} / 3\right) V^{(1 / 3)\left(D_{s}-3\right)} \text {. }
$$

When $D_{s}=2$, Eq. (4) reduces to give the $1 / r$ dependence that is typically assumed for capillary pressure [6-8,24].

\section{MOISTURE CONTENT AND CAPILLARY PRESSURE}

The moisture content within a two-phase system at equilibrium can be written as $V_{w}=\int V d N$, where $V_{w}$ is the fluid volume within a REV, $V$ is the volume of a single filled pore of a given size, $N$ is the number of those pores within the $\mathrm{REV}$, and the integral is over the applicable size range. If the pore volume is distributed in a fractal manner, we can follow Perrier et al. [8] who used the cumulative distribution function $N r(l>L)=F L^{-D_{v}}$ to determine total pore volume, where $L$ is taken to be the radius that corresponds to $V, F$ is a constant, and $D_{v}$ is the pore volume fractal dimension. Noting that $L \propto V^{1 / 3}$ it can then be shown that

$$
V_{s}-V_{w}=\left(F D_{v}\right) /\left(3-D_{v}\right)\left(V_{\max }^{\left(3-D_{v}\right) / 3}-V^{\left(3-D_{v}\right) / 3}\right),
$$

where $V_{s}$ is the saturated water volume within a REV, $V$ is the volume of the smallest pore filled, and $V_{\max }$ is the volume of the largest. Dividing by the volume of the REV and setting $V_{0}=\left(F D_{v}\right) V_{\max }^{(3-D v) / 3} /\left(3-D_{v}\right)$ we get

$$
\theta_{s}-\theta=V_{0} / V_{\mathrm{REV}}\left[1-\left(V / V_{\max }\right)^{\left(3-D_{v}\right) / 3}\right] \text {, }
$$

which is essentially the relation derived by Perrier et al. [8]. Substituting from Eq. (4) we then get the relationship between media saturation and capillary pressure:

$$
\theta_{s}-\theta=V_{0} / V_{\mathrm{REV}}\left[1-\left(P_{s} / P\right)^{\lambda}\right] \text {, }
$$

where $\lambda=\left(3-D_{v}\right) /\left(3-D_{s}\right)$, and $P$ and $P_{s}$ are the saturated and unsaturated capillary pressures. This is applicable to a 
two phase system with wetting and nonwetting fluids. As with previous power-law formulations relating capillary to moisture content, Eq. (7) is likely to breakdown near saturation.

The results of Rieu and Sposito [7] and Tyler and Wheatcraft [6] can be obtained as special cases of Eq. (7). When the size of the smallest pore filled within the REV, $V_{\text {min }}$, is equal to $0, V_{0} / V_{\mathrm{REV}}=\theta_{s}$ and Eq. (7) also gives the well-known relationship presented by Brooks and Corey [1], where $\theta / \theta_{s}=\left(P_{s} / P\right)^{\lambda}$. This equation is sometimes written as $\left(\theta_{s} \theta\right) /\left(\theta_{s}-\theta_{r}\right)=\left(P_{s} / P\right)^{\lambda}$, where the left-hand side is termed the "reduced water content" with $\theta_{r}$ being the "residual water content" (i.e., the value below which vapor transport is required to reduce the water content further). The residual water content effectively represents the percolation threshold for the medium. The choice of plotting $P_{s} / P$ against reduced water content stems less from the physics of the phenomena than because of a historical convention that was first used by Burdine ([25] and [26], p. 479) to force relationships for hydraulic conductivity to zero at the percolation threshold.

\section{CONCLUSIONS}

Many studies have measured the pore volume fractal dimension directly and the values obtained to date indicate that $3-D_{v}<1$ [27]. However, values of $\lambda$ that are larger than this, and indeed greater than 2 , have long been known to occur, indicating $\lambda=3-D_{v}$ is insufficient $[1,27,28]$. By considering a porous system with distinct pore surface and pore volume fractal dimensions we have derived a more general form of $\lambda=(3-D) /\left(3-D_{s}\right)$. When $D_{s}=2$, the analysis reduces to the standard relationship, as expected, and when $D_{s}>2$ it allows for a larger value of the exponent than 3 $-D_{v}$. The results show that capillary pressure data alone are insufficient to establish the fractal nature of a pore space and that data on the pore surface fractal dimension are needed as well.
[1] R. H. Brooks and A. T. Corey (unpublished).

[2] M. Rieu and G. Sposito, Soil Sci. Soc. Am. J. 55, 1239 (1991).

[3] P. G. de Gennes, in Physics of Disordered Materials, edited by M. Daoud (Plenum, London, 1985).

[4] H. T. Davis, Europhys. Lett. 8, 629 (1989).

[5] J. C. Melrose, paper SPE 18331, presented at the 63rd Annual Conference of the Society of Petroleum Engineers, Houston, Texas (1988).

[6] S. W. Tyler and S. W. Wheatcraft, Water Resour. Res. 26, 1047 (1990).

[7] M. Rieu and G. Sposito, Soil Sci. Soc. Am. J. 55, 1231 (1991).

[8] E. Perrier, M. Rieu, G. Sposito et al., Water Resour. Res. 32, 3025 (1996).

[9] J. T. Chen, L. J. Pyrak-Nolte, D. D. Nolte et al., Geophys. Res. Lett. 31, L08502 (2004).

[10] M. R. Deinert, J. Y. Parlange, and K. B. Cady, Phys. Rev. E 72, 041203 (2005).

[11] A. N. Anderson, A. B. McBratney, and J. W. Crawford, in Advances in Agronomy, edited by D. L. Sparks (Academic Press, San Diego, 1998), Vol. 63, p. 1.

[12] P. Pfeifer, D. Avnir, and D. Farin, Surf. Sci. 126, 569 (1983).

[13] P. Pfeifer and D. Avnir, J. Chem. Phys. 79, 3558 (1983).

[14] D. Gimenez, R. R. Allmaras, E. A. Nater et al., Geoderma 77, 19 (1997).
[15] F. Stallmach, C. Vogt, J. Karger et al., Phys. Rev. Lett. 88, 105505 (2002).

[16] A. Dathe and M. Thullner, Geoderma 129, 279 (2005).

[17] J. P. Hansen and A. T. Skjeltorp, Phys. Rev. B 38, 2635 (1988).

[18] F. Bartoli, R. Philippy, M. Doirisse et al., J. Soil Sci. 42, 167 (1991).

[19] A. N. Anderson, A. B. McBratney, and E. A. FitzPatrick, Soil Sci. Soc. Am. J. 60, 962 (1996).

[20] D. Weitz, J. P. Stokes, R. C. Ball et al., Phys. Rev. Lett. 59, 2967 (1987).

[21] S. M. Hassanizadeh and W. G. Gray, Adv. Water Resour. 13, 169 (1993).

[22] B. Mandelbrot, The Fractal Geometry of Nature (Freeman, New York, 1983).

[23] B. Mandelbrot, D. E. Passoja, and A. J. Paullay, Nature (London) 308, 721 (1984).

[24] Y. F. Xu and P. Dong, Chaos, Solitons Fractals 19, 327 (2004).

[25] N. T. Burdine, Petr. Trans. AIME 198, 71 (1953).

[26] J. Bear, The Dynamics of Fluids in Porous Media (Dover, Mineola, NY, 1988).

[27] R. Havenkamp, F. J. Leij, C. Fuentes et al., Soil Sci. Soc. Am. J. 69, 1881 (2005).

[28] R. Haverkamp and J. Y. Parlange, Soil Sci. 142, 325 (1986). 